Revue internationale P.M.E.

Économie et gestion de la petite et moyenne entreprise

Revue

internationale

PME

\title{
Les petites entreprises dans l'économie de l'URSS
}

\section{A.V. Avilova}

Volume 5, numéro 1, 1992

URI : https://id.erudit.org/iderudit/1008131ar

DOI : https://doi.org/10.7202/1008131ar

Aller au sommaire du numéro

Éditeur(s)

Presses de l’Université du Québec

ISSN

0776-5436 (imprimé)

1918-9699 (numérique)

Découvrir la revue

Citer cet article

Avilova, A. (1992). Les petites entreprises dans l'économie de l'URSS. Revue internationale P.M.E., 5(1), 27-41. https://doi.org/10.7202/1008131ar

\section{Résumé de l'article}

Cet article traite de la situation des petites entreprises (PE) en URSS, un sujet d'étude encore nouveau dans le spublications économiques soviétiques. Après une description des particularités de ces entreprises par rapport à celles des autres pays d'Europe orientale, l'auteure distingue trois types de PE dont la dynamique reflète certaines structures de marché qui ont vu le jour en URSS et qui se développent assez rapidement.

Le plus important type représente les PE traditionnelles fonctionnant hors marché. Ces entreprises sont incluses dans la structure industrielle monopolisée. Le deuxième type, pouvant être qualifié de quasi-marché, est né pendant la réforme et suit révolution des rapports de propriété. Le troisième type, le plus nouveau et le plus proche de la version occidentale de la petite entreprise, évolue sur le marché libre.

L'auteure montre que l'État n'a pas de stratégie de création d'un secteur autonome de petites entreprises et que la tendance prédominante consiste plutôt en une " privatisation spontanée ». Enfin, l'auteure dresse un tableau des conditions nécessaires au développement de la petite entreprise et de l'orientation des changements en cours.
Ce document est protégé par la loi sur le droit d'auteur. L'utilisation des services d'Érudit (y compris la reproduction) est assujettie à sa politique d'utilisation que vous pouvez consulter en ligne.

https://apropos.erudit.org/fr/usagers/politique-dutilisation/ 


\title{
Les petites entreprises dans l'économie de l'URSS
}

\author{
A.V. AVILOVA \\ Imemo**
}

\begin{abstract}
RÉSUMÉ
Cet article traite de la situation des petites entreprises (PE) en URSS, un sujet d'étude encore nouveau dans le spublications économiques soviétiques. Après une description des particularités de ces entreprises par rapport à celles des autres pays d'Europe orientale, l'auteure distingue trois types de PE dont la dynamique reflète certaines structures de marché qui ont vu le jour en URSS et qui se développent assez rapidement.

Le plus important type représente les PE traditionnelles fonctionnant hors marché. Ces entreprises sont incluses dans la structure industrielle monopolisée. Le deuxième type, pouvant être qualifié de quasi-marché, est né pendant la réforme et suit l'évolution des rapports de propriété. Le troisième type, le plus nouveau et le plus proche de la version occidentale de la petite entreprise, évolue sur le marché libre.

L'auteure montre que l'État n'a pas de stratégie de création d'un secteur autonome de petites entreprises et que la tendance prédominante consiste plutôt en une * privatisation spontanée . Enfin, l'auteure dresse un tableau des conditions nécessaires au développement de la petite entreprise et de l'orientation des changements en cours.
\end{abstract}

\section{ABSTRACT}

This article discusses the situation of small businesses in the USSR, a still recent subject in Soviet economic publications. Following a description of the characteristics of these businesses in relation to those in other European

* Cet article a été écrit, pour l'essentiel, avant les événements des 19-21 août 1991. Les matériaux factuels et les statistiques qui y figurent concernent la totalité du territoire de l'ex-URSS.

** Dr Agnessa Avilova Ph.D. en économique de l'Institut des relations intemationales de Moscou, spécialiste en économie de l'Europe du Sud. Quelques publications récentes : L'économie de l'Espagne (1978) The Countries of Southern Europe in Contemporary World (1989). 
countries, the author identifies three types of $S B$ whose dynamic reflects certain market structure initiated in the USSR which are developing fairly rapidly. The most important type represents traditional SBs working outside the market. These enterprises are part of the monopolized industrial structure. The second type, which could be called quasi-market, were born during the reform and follow the evolution of ownership return. The third type, the newest and the closest western version of small business, develops in a free market.

The author shows that the State has no strategy for the creation of an independent sector of small businesses and that the prevalent tendancy is one of spontaneous privatization. In conclusion, the author presents an overall picture of the conditions necessary for the development of small business and the direction present changes are taking.

\section{RESUMEN}

Este artículo discute la situacion de las pequeñas empresas (PE) en URSS, un tema de estudio todavia nuevo dentro de las publicationes económicas sovieticas. Despues de una descripcion de las particularidades de éstas empresas en comparasión a las empresas de los otros paises de Europa oriental, la autora distingue tres tipos de PE en las cuales la dinámica refleja cierta estructura del mercado que han nacido en las URSS y que se derarrollan rápidamente.

El tipo más importante de empresas son las tradisionales que funcionan fuera del mercado. Estas empresas estan incluidas dentro de la estructura industrial monopolisada. El segundo tipo, puede ser calificado de casi-mercado, es nacida durante la reforma ysigue la evolución de las reparticiónes de la propriedad. El tercer tipo, el mas reciente y el mas próximo de la version occidental de la PE, evoluciona dentro de un mercado libre.

La autora muestra que el estado no tiene una estragia de creacion de un sector autónomo de PE y que la tendencia predominante consiste preferentemente en una « privatisación espontánea ". Enfin, la autora dirige un cuadro completo de las condiciónes necesarias al desarrollo de la PE y de la orientación de los cambios en curso.

\section{La spécificité du problème}

Par rapport aux autres pays d'Europe orientale, la situation des petites entreprises (PE) dans l'économie de l'URSS se caractérise par deux particularités.

Premièrement, par rapport aux pays industrialisés, la part de PE dans la structure de la production et de l'emploi y est anormalement faible. Cela est dû au fait que l'économie soviétique s'est développée pendant deux décennies d'industrialisation forcée et ce, dans un contexte où prédomine la propriété d'État. Le développement normal des petites entreprises - comme des entre- 
prises en général - a cessé avec la disparition du marché libre. Aussi, le monopolisme et la superconcentration restent les principales caractéristiques de notre système économique.

Deuxièmement, la réforme économique entamée en 1986 n'a pas encore conféré aux petites entreprises le rôle qu'elles pourraient jouer pour faire revenir le pays à l'économie de marché. La nouvelle législation, fruit de longues discussions et de lutte politique, est pleine de contradictions et demeure très limitée. Ce que la petite entreprise a obtenu à ce jour, elle le doit principalement à elle-même, en se développant malgré une piètre création de conditions sociales et économiques appropriées.

L'étude du problème des PE sur le plan national ne fait que commencer dans nos publications économiques. Jusqu'au milieu des années 80 , la notion de « big is beautiful » prédominait dans la théorie et la pratique de l'économie soviétique : les entreprises créées devaient avoir, au minimum, une taille européenne. La crise a contraint les économistes à revoir cette approche, rendant évidente, pour beaucoup d'entre eux, la nécessité de démembrer la production et de centrer l'attention des spécialistes sur les potentialités des petites entreprises de l'Occident. Les tentatives pour imiter cette expérience ont cependant montré le caractère très limité de son applicabilité dans une économie sans marché libre. À présent, la tâche des économistes est d'élaborer une conception globale et de faire des recommandations pratiques pour orienter la politique structurelle actuelle vers la mise en place de nouveaux mécanismes économiques dans le pays.

Le développement de la petite entreprise en URSS avait été interrompu à la fin des années 20 , alors que ce secteur employait 4 millions de personnes - $58 \%$ des salariés de l'industrie - et fournissait $25 \%$ de la production industrielle. Les trois quarts de cette main-d'œuvre avaient conservé des liens avec la campagne et la grande partie était des coopérateurs. Au cours de la collectivisation (qui démantela l'ancienne structure agricole) et de l'industrialisation, la petite production a subi des dommages importants et en 1937, sa part dans la production industrielle était déjà tombée à 5,6\%. Dans les années 60, on étatisait les coopératives artisanales qui comptaient 200000 entreprises employant 1,8 million de personnes et travaillant principalement pour la consommation. $\grave{A}$ partir de ce temps, la propriété d'État régnera sans partage.

Dans le rapport « L'économie de l'URSS : conclusions et recommandations » préparé par une équipe d'experts internationaux en vue de la Conférence des « Sept » à Londres en avril 1991, nous lisons : « Le secteur industriel est constitué presque entièrement d'énormes compagnies publiques. Le nombre moyen de personnes travaillant dans chacune des 47000 entreprises du pays dépasse 800 ; approximativement 30 à $40 \%$ du volume des produits sont fabri- 
qués par un seul producteur [...] $75 \%$ des commandes et des ressources émanent de l'État ; quant aux prix, ils sont fixés par voie administrative et ne sont pas régulés par le marché [...] La propriété individuelle dans le secteur industriel se limite à l'artisanat qui ne donne pas lieu à du salariat ».

Ces chiffres ne fournissent pas encore une image complète du niveau de prédominance de la grande production. On doit ajouter que les trois quarts de la main-d'œuvre industrielle se concentrent dans les entreprises employant plus de 6000 personnes, concentration qui s'est accentuée encore tout récemment. La pyramide de la structure industrielle en URSS est inversée : les entreprises dont le personnel ne dépasse pas 100 salariés ne constituent que 3,5\% du nombre total d'entreprises (contre près de $90 \%$ aux États-Unis), les 500000 petites et moyennes entreprises de toutes les branches ne représentent pas plus de $20 \%$ du PNB (voir annexe); tout comme est inversée aussi la structure sectorielle de l'économie : seulement $25 \%$ des entreprises industrielles de la Fédération de Russie où 6 à $8 \%$ des unités de production sont spécialisées dans la fabrication des biens de consommation.

À l'heure actuelle, dans un contexte de crise profonde et de pénurie dans la quasi-totalité des produits et des services, le rétablissement de la petite production est une nécessité pressante. Bien entendu, il s'agit non seulement de la renaissance des secteurs traditionnels, mais aussi de la création de nouvelles productions modernes du point de vue technique. La petite entreprise pourrait rapidement corriger de nombreuses lacunes dans la demande, lesquelles sont engendrées par les désordres monstrueux observés dans la structure industrielle, ce qui aiderait à résorber le chômage qui a déjà pris des proportions inquiétantes dans certains régions; la PE pourrait aussi contribuer à surmonter le monopolisme, principal vice du système en place, soit favoriser le progrès technique dans le pays, éliminer les "goulots d'étranglement » de l'infrastructure (surtout dans le domaine du prêt-bail et autres services industriels) et enfin, stimuler les affaires dans les petites et moyennes villes. Les petites entreprises assurent près de $40 \%$ des services fournis à la population et, dans ce domaine, la demande dépasse l'offre de 3 à 3,5 fois.

D'après les spécialistes, l'économie de marché dans notre pays commencera à fonctionner relativement normalement quand quelque 3 millions de petites unités de production y auront été créées. Le projet gouvernemental publié au mois de juillet 1991 prévoit qu'il en sera créé 1,5 à 2 millions en deux ans ; mais même si ce programme est mené à bien, la densité du réseau des PE restera de 4 à 6 fois moins élevée qu'aux États-Unis, au Japon ou en RFA. Le démembrement des entreprises existantes n'assurera la formation que de $30 \%$ à $35 \%$ de ce réseau ; le reste devra être entièrement créé. 
Ce qui précède ne donne toutefois qu'une idée générale des caractéristiques et de l'ampleur des problèmes. Examinons maintenant la situation de façon plus concrète.

\section{Trois types de PE}

Dans le contexte de l'économie soviétique, le terme PE n'a pas la même signification qu'en Occident. L'économie soviétique dans son ensemble reste axée sur la distribution ; les entreprises ne sont pas libres de réaliser toute leur production; de nombreuses activités, normales en Occident, sont interdites par la loi. Mais certaines structures de marché ont déjà vu le jour et se développent assez rapidement. Dans l'optique des tâches principales de la réforme - la transition au marché - on peut distinguer trois types de PE, dont la dynamique reflète à sa façon ce processus.

Le premier type - le plus important - représente les PE traditionnelles fonctionnant hors marché ; celles-ci sont incluses dans la structure industrielle monopolisée et représentent un des innombrables « boulons » de celles-ci. C'est habituellement une entreprise mal équipée et employant une main-d'œuvre nombreuse et peu qualifiée. En voici quelques exemples : dans les petites entreprises du complexe agro-industriel d'Ukraine, la productivité du travail est 2,5 fois inférieure à la productivité moyenne dans la branche industrielle, elle l'est de 2,3 fois dans l'industrie légère, de 7 fois dans la métallurgie, de 4,5 fois dans l'industrie chimique ; dans la région minière de Donetsk, la rentabilité des PE est de 2 à 4 fois, parfois de 10 fois moindre que celle des grandes unités de production. Il s'agit d'entreprises créées par le système bureaucratique et autoritaire et qui en possèdent tous les vices, dont le principal est sa non-viabilité. Avec le passage au marché libre, ces PE devront se moderniser ou disparaître.

Le deuxième type, né pendant la réforme, est relié à l'évolution des rapports de propriété. Ce type peut être qualifié de quasi-marché : bien qu'il présente tous les signes de la microstructure autonome de marché, il ne peut pas transformer le milieu environnant et il est contraint de respecter des règles de jeu où les normes administratives de conduite l'emportent sur les normes concurrentielles. Il comprend premièrement les PE publiques, formellement indépendantes, mais évoluant dans le cadre de complexes de production plus grands ; deuxièmement, on compte des entreprises de l'économie dite alternative, principalement orientées sur le secteur de l'État. 
Comment s'établiront les rapports entre ce type de PE et la structure monopolisée ? Prenons un premier cas : celui d'une petite unité formellement détachée d'une grande entreprise.

Il s'agit ordinairement d'une "unité naturelle » avec de nombreuses productions annexes couvrant les besoins de la production principale. Selon les estimations, la spécialisation de cette entreprise et la réduction du personnel permettront de réduire de $15 \%$ le prix de revient et d'accroître les bénéfices de 35 à $40 \%$. Si, auparavant, le démembrement était ordinairement banni, aujourd'hui l'exacerbation de la crise pousse les chefs d'entreprise à remettre en question cette situation; ils acceptent la création de nouvelles PE avec ces productions annexes, tout en les maintenant sous leur contrôle en tant que « fondateurs". Ce statut leur confère le droit d'élaborer les statuts de la nouvelle entreprise et les conditions du bail, de nommer et de destituer son directeur, d'utiliser cette PE pour la fabrication de certains types de produits et de se « payer » grâce aux avantages procurés par l'État.

Dans ce cas, l'indépendance de la PE est éphémère ; elle n'a ni le droit de sortir du contrôle du fondateur, ni de choisir la forme de propriété de son capital. Même si elle donne l'impression d'introduire les principes du marché dans l'économie, en réalité cette façon de faire sert à masquer le maintien du monopole d'État et de la concentration de la production.

En laissant de côté les cas de démembrement des firmes, ce qui répond effectivement aux impératifs économiques, remarquons que de nombreux fondateurs sont enclins à vivre aux dépens des PE qu'ils créent. Ainsi, lors du détachement d'une coopérative de réparation, on oblige celle-ci à réaliser tous les travaux dont le fondateur a besoin, et ce, à des tarifs plus élevés. Un bureau d'études coopératif, qui emploie par cumul des collaborateurs de l'organisation qui l'a constitué, a le droit de décider quelles autres commandes il exécutera en tant que coopérative et à des tarifs plus élevés. Ainsi des subdivisions du pouvoir exécutif local constituées en PE accordent contre paiement des services qui auparavant étaient gratuits.

Évidemment, tout cela n'est qu'une imitation du marché. Mais le principal préjudice causé par cette pratique consiste en ce que le truchement du mécanisme de crédit permet le transfert sur une grande échelle de monnaie scripturale en monnaie fiduciaire, ce qui est une des causes de la déstabilisation de la circulation monétaire dans le pays ces dernières années.

À la différence des PE d'État, les entreprises de l'économie dite alternative sont juridiquement complètement indépendantes. Ce secteur doit son existence à une série de lois adoptées entre 1986 et 1990 pour légaliser l'entreprise ; lois touchant le travail individuel (remplacée par la loi sur les principes géné- 
raux de l'entreprise en URSS), la coopération, les principes du bail et les mesures à prendre en vue de créer et de développer les petites entreprises (arrêté du Conseil des ministres en date du 8 août 1990). L'économie dite alternative est constituée pour l'essentiel de petites entreprises. Cela dit, non seulement le " travail individuel », mais aussi la plupart des coopératives sont, sous une forme dissimulée, une entreprise privée. Une étude portant sur des dizaines de coopératives en Sibérie a montré, entre autres, que pour neuf sur dix d'entre elles le capital initial avait soit appartenu à un fondateur, soit été apporté par une banque. Le statut de la PE a pour la première fois légalisé l'utilisation du salariat dans les entreprises privées et leur a accordé divers avantages fiscaux ${ }^{1 .}$

L'apparition de ces nouvelles formes d'activité a entrâné un changement notable dans la balance générale des liens économiques au profit des structures de marché. Au début de 1991, ce secteur fournissait déjà $15 \%$ de la production nationale. Les coopératives ont connu un essor particulièrement rapide ; 299400 ont été enregistrées (245 400 fonctionnent réellement); elles emploient 6,1 millions de personnes (dont 1,9 million par cumul) ; la valeur de leur production est passée de 330 millions à 70 milliards de roubles en trois ans.

En quelques mois, 400000 entreprises ont obtenu le statut de PE. Sept cent mille personnes, dont 400000 par cumul, étaient concernées par le régime du travail individuel.

Cependant, les conditions dans lesquelles l'activité de ce secteur a été placée dès le début l'ont empêché d'acquérir la « masse critique » indispensable pour entraîner l'ensemble de l'économie sur la voie du marché. Selon V. Legler, un spécialiste de la question, le potentiel des coopératives ne peut être

1. Le critère permettant à une entreprise de revendiquer ce statut est l'importance numérique de son personnel : jusqu'à 200 salariés dans l'industrie et le bâtiment, jusqu'à 100 dans le domaine de la recherche-développement, jusqu'à 50 dans les autres branches industrielles de la production matérielle, jusqu'à 25 dans les services (excepté le commerce de détail), jusqu'à 15 dans le commerce de détail. Le deuxième critère, la valeur de la production, doit être différencié selon les républiques.

Les PE sont exemptes d'impôts sur les bénéfices les deux premières années d'activité à condition de transformer des matières premières agricoles, de produire des biens de consommation, de réaliser des travaux de réparation et de construction, de produire des matériaux de construction et d'introduire des innovations techniques. Pour les autres types d'activité, les impôts sont réduits de $25 \%$ la première année et de $50 \%$, la deuxième. Des avantages sont également accordés aux entreprises qui emploient des invalides et des retraités. Ne sont pas soumis à l'impôt les fonds utilisés pour la modernisation et l'extension du capital fixe, le recours à des technologies nouvelles et la formation professionnelle. 
pleinement déployé parce que dans l'économie soviétique, trop peu de ressources sont réalisées en espèces. Cela oblige les coopératives à s'intégrer dans le système des commandes publiques et de l'approvisionnement en matériel et technique. Près de 4 coopératives sur 5 réalisent des programmes planifiés ; $60 \%$ des équipements utilisés sont loués à l'État. Aussi n'est-il pas étonnant que l'efficacité de marché des coopératives soit si faible : leur production ne représente pas plus de $2 \%$ du commerce de détail.

Le niveau de la fiscalité est une autre raison qui limite les possibilités de manœuvre des PE. L'impôt sur les bénéfices est égal à $35 \%$; les autres impôts, fluctuants, peuvent atteindre au total près de $90 \%$. L'État prélève aussi $80 \%$ des rentrées en devises convertibles. Pour le statut de la PE, on fixe le niveau plafond de la rentabilité à $50 \%$, le reste est versé au budget. Le programme de privatisation récemment élaboré par Moscou comporte l'institution de 16 types d'impôts municipaux.

Dans les domaines où les coopératives concurrençaient le secteur public, les autorités les ont évincées en réduisant la portée de la législation au moyen d'instructions ministérielles. C'est ainsi, par exemple, qu'un terme a été mis à l'activité médiatrice des coopératives en matière de commerce.

Il est difficile de dire quelle partie de l'économie alternative était au début réellement alternative ; à l'heure actuelle, 2 à 10 de ces structures, utilisées habituellement pour obtenir des revenus supplémentaires, sont reliées à chaque grande entreprise ou établissement public. Mais c'est justement dans ce « bouillon de culture » qu'est né le troisième type de PE, celui du marché libre, le plus proche de la version occidentale de la petite entreprise. S'y rapporte ou tend vers lui la partie du secteur non public qui, pour une raison quelconque et grâce à une moins grande dépendance aux matières premières, ainsi qu'à un meilleur accès aux ressources matérielles ou aux sources de financement, possède une véritable liberté d'entreprendre.

L'existence de ce nouveau type est attestée par la création d'organisations telles que 1'Union moscovite des entrepreneurs privés (en mars 1990: un millier de membres), l'Union russe des propriétaires privés (en octobre 1990 : plus de 5000 membres) ou la bourse Fermer (en septembre 1991), qui a l'intention de travailler avec 30000 paysans indépendants de la Fédération de Russie.

La frontière entre le deuxième et le troisième types de PE n'est pas statistiquement définissable ; elle a tendance à se déplacer au profit du dernier type à mesure que l'économie de marché s'étend dans l'économie générale. Cette extension dépend au premier chef de la modernisation de l'infrastructure. Au mois de septembre 1991, près de 370 bourses de commerce et de matières premières (la première a été ouverte à Moscou en mai 1990), 1500 banques 
commerciales et coopératives, des milliers de bureaux de courtage, des dizaines de sociétés d'audit, d'innombrables écoles de gestion fonctionnent dans le pays. Les ventes aux enchères de disponibilités deviennent régulières. Sur la base des centres régionaux du Gossnab (Comité d'État pour l'approvisionnement), on implante un réseau informationnel commercial. Dans le contexte de la désagrégation rapide de l'ancien système économique et des mécanismes de gestion, ce sont précisément ces structures, dans le cadre desquelles le capital d'État et le capital privé opèrent conjointement, qui soutiennent l'activité économique. Selon les estimations, en 1992, près de la moitié du pétrole et des métaux, $30 \%$ des céréales et autres produits alimentaires seront vendus par le truchement des bourses.

Il est significatif que l'infrastructure de marché s'impose « de sa propre autorité », sans attendre l'élaboration et l'entrée en vigueur de la nouvelle législation.

Comment les petites entreprises se sentent-elles dans cette situation? Quelle place leur appartient-il dans le processus des changements à l'étape actuelle de la réforme économique en URSS ?

\section{Les problèmes des petites entreprises. La privatisation}

L'analyse des derniers travaux scientifiques et des articles de presse permet de résumer ces problèmes de la façon suivante.

L'essor de la petite entreprise est surtout entravé par la monopolisation du marché naissant, monopolisation due à l'absence d'accès libre et égal aux sources de matières premières, au capital et à la main-d'œuvre ; il est limité aussi par le sous-développement de ce marché, aggravé par l'interdiction d'achat et de vente de terrains et autres biens immobiliers. Le système de crédit n'est pas adapté au financement des PE. Le pays possède un infime réseau de crédit commercial et de sociétés d'assurances, sans parler des « compagnies à risque » ou des garants spéciaux pour la petite entreprise, telles les associations régionales ou les comités antimonopoles.

L'État n'a pas de programmes spéciaux de développement et d'aide à la petite entreprise, ce qui introduirait un système de garanties et autres avantages, analogue à celui qui existe partout en Occident. Les organes d'autogestion territoriale, avec des institutions acceptant la participation des petites entreprises au règlement des problèmes locaux, ne sont pas développés. La politique fiscale 
à l'égard des PE est impitoyable et nuit systématiquement à l'innovation et à l'accumulation du capital. Pour ces diverses raisons, l'indépendance économique des petites entreprises et ses possibilités de manœuvre et d'investissements à long terme sont limitées.

La législation dans ce domaine est très imparfaite. Il existe trois groupes de documents se rapportant aux niveaux fédéral et local, mais ils sont mal articulés. Il est impossible d'y trouver une définition précise du statut de la PE et une liste des activités autorisées, ce qui se traduit dans la pratique par des interdictions arbitraires. Sont absents aussi le principe de la délimitation entre les structures publiques et non publiques et les dispositions régularisant les rapports entre les PE et les grandes entreprises. D'où le « caractère nébuleux du statut juridique » (dont il est fait état plus haut dans le rapport des experts internationaux) qui, apparemment, convient parfaitement à ceux qui l'ont élaboré.

L'absence d'une protection juridique sûre et l'incertitude du lendemain incitent de nombreuses PE à rechercher le profit immédiat, ce qui entraine toutes sortes d'abus. L'ancienne culture de la petite entreprise a disparu ; quant à la nouvelle, elle prend corps dans des conditions défavorables.

Ce qui précède conduit à une conclusion évidente : l'État ne possède pas de stratégie de création dans le pays d'un secteur autonome de petites entreprises qui répondrait aux objectifs de la réforme économique proclamée.

Au lieu de cela, la tendance prédominante depuis à peu près le début de l'année 1990 est la " privatisation spontanée », sorte de version "nomenklaturienne » de transition au marché. Il s'agit ici de la création intensive, par les ministères sectoriels et les complexes industriels, d'associations, de consortiums ou autres groupements indépendants de l'État qui, sous prétexte de passer à l'économie de marché, s'attribuent le droit de posséder les biens des entreprises qui leur sont subordonnées. Rompant formellement avec le système administratif, ces formations nouvelles s' emparent des biens publics jadis dépersonnalisés, mais qui acquièrent aujourd'hui des propriétaires concrets ; le régime du monopole est maintenu et se fortifie sous le mot d'ordre du « marché ».

Les capitaux du PCUS et des organes du pouvoir exécutif sont transférés à ces associations et consortiums. Selon une déclaration faite par les dirigeants du « secteur alternatif » à la veille justement du putsch d'août, par ce canal, la nomenklature a déjà réussi à s'approprier la moitié des entreprises de services. Au début de 1991, on dénombrait dans le pays 1420 associations économiques, 126 consortiums, 156 groupements intersectoriels fédéraux et territoriaux, 102 grandes unions d'entreprises, plus de 1200 sociétés par actions. Les possibilités financières de cette nouvelle élite et celles du secteur alternatif ne sont pas comparables : le capital des 148 banques commerciales desservant cette élite 
s'élève à 4,5 milliards de roubles alors que les 108 banques coopératives liées au secteur alternatif ne disposent que de 115 millions de roubles, soit 2,5\% seulement de l'ensemble du fonds statutaire des établissements bancaires du secteur non public.

L'accaparement des biens par les appareils de l'État et du PCUS empêche la modernisation de l'économie. Comme l'atteste le $D^{r}$ Stanley, spécialiste américain de la privatisation et de l'actionnariat, " la plupart des gestionnaires des anciennes entreprises publiques soviétiques ignorent ce que c'est que la gestion efficace et ce qu'il faut faire pour cela ». Les PE qui voient le jour au sein de cette structure n'en sont qu'un élément fonctionnel et, sous leur aspect actuel, elles ne sont pas à même de jouer un rôle " modernisateur » - en ouvrant l'accès à la propriété ou en contribuant à la création dans le pays d'une « classe moyenne nouvelle ». Ces PE sont utilisées par l'élite comme moyen de mimétisme social ou d'enrichissement.

Cette fausse « désétatisation » est caractéristique aussi de la majorité des toutes nouvelles organisations représentatives des milieux d'affaires, dont la création a été décidée d'en haut. Ces organisations sont chapeautées par l'Union scientifico-industrielle, dont font partie tous les « grands » de l'industrie soviétique, en compagnie des organisations scientifiques, commerciales et financières pilotes du pays (la propriété d'État y entre pour $90 \%$ ). La Fédération pour le développement et le soutien de la petite entreprise, au sein de laquelle les PE du secteur public prédominent et dont la direction comporte des représentants du Gosplan et du Conseil des ministres, ne fait pas exception.

La décentralisation politique et économique peut apporter certaines corrections dans le cours de la réforme. Depuis cinq ans, la part de la production des entreprises relevant des républiques ou du local en URSS est passée de 4,5 à $37 \%$. Après le 21 août, le processus est entré dans une phase nouvelle, celle où la privatisation va devoir se déployer au niveau régional. Des programmes assez radicaux, dépassant les clauses de la Loi fédérale sur la privatisation votée le 8 août 1991, ont été adoptés en Russie, au Kazakhstan, en Arménie, ainsi qu'à Moscou. Cependant, seul le temps permettra de juger des éventuels progrès réels dans cette direction, tout comme de leurs résultats sociaux.

\section{Le milieu social}

Cela nous place devant la question de la présence dans le pays des conditions nécessaires au développement de la petite entreprise. On ne saurait ici fournir une réponse univoque : la situation est hétérogène et évolue avec une telle 
rapidité que les données des sondages risquent de devenir caduques avant même la fin de l'analyse. Aussi détacherons-nous seulement quelques aspects permettant de brosser un tableau d'ensemble et de discerner l'orientation des changements en cours.

La base sociale pour le développement de la petite entreprise est restreinte d'emblée, surtout en raison de l'insuffisance d'épargnes pouvant être utilisées durant la privatisation. Ainsi, en Biélorussie les particuliers et les collectifs ne peuvent acheter à l'État plus de $7 \%$ de ses fonds fixes ; cet indice tombe entre 3 à $5 \%$ dans la Fédération de Russie (si l'on ne tient pas compte des investissements émanant de l' «économie parallèle »). Apparemment, la situation dans l'ensemble du pays n'est pas meilleure : selon les données d'un sondage, plus de la moitié des personnes souhaitant monter leur propre affaire n'en ont pas les moyens. Mais il existe aussi des raisons sociopsychologiques, sérieuses elles aussi. Les sentiments hostiles à l'entreprise sont répandus, une grande partie de la population est paupérisée : ne possédant pas de patrimoine, elle a perdu tout désir de jouer un rôle économique autonome. Les ouvriers non qualifiés, qui représentent jusqu'à $30 \%$ de l'ensemble de la corporation, considèrent souvent le paternalisme de l'État comme un dû et se montrent indifférents ou hostiles visà-vis de la réforme.

Bien évidemment, la situation n'est pas la même partout. Ce n'est pas un hasard si la réforme a été amorcée dans les Républiques baltes (désormais États indépendants). Celles-ci détenaient le leadership en Union soviétique dans le domaine de la production coopérative : en 1990, la valeur de la production réalisée par les coopératives a atteint 760 roubles par habitant en Lettonie, 617 en Estonie, 394 en Arménie, alors qu'elle n'a été que de 47 roubles en Azerbaïdjan, 72 en Kirghizie, 88 en Turkménie (moyenne nationale : 232 roubles). Cet écart du simple au décuple atteste des différences énormes qui existent dans les traditions économiques de la population, ce qui aura assurément une incidence sur le développement ultérieur de la petite entreprise.

Cependant, il serait erroné de sanctifier le rôle de ces facteurs aujourd'hui, quand la crise allant en s'aggravant incite toutes les couches de la société et les régions à chercher un nouveau modèle économique. Les esprits évoluent assez rapidement. À la question: «Êtes-vous pour ou contre le développement de l'entreprise privée ? " posée avec un intervalle de 13 mois (novembre 1989 et décembre 1990) par le Centre fédéral d'étude de l'opinion publique, les réponses ont été celles-ci : pour, respectivement 31 et $44 \%$; contre, 42 et $26 \%$; indécis, 42 et $30 \%$. En décembre 1990, le plus grand nombre de partisans de la petite entreprise se trouvaient parmi les jeunes âgés de 24 ans et moins (55\%), les personnes possédant une instruction supérieure $(57 \%)$ et les personnes aux revenus élevés $(70 \%$ ). Au niveau des régions, cet indice était de $74 \%$ parmi 
la population de la Géorgie, de $70 \%$ en Estonie, de $53 \%$ en Biélorussie, de $43 \%$ au Kazakhstan et de $42 \%$ en Fédération de Russie.

L'image sociale du secteur privé de l'économie change. À l'été 1991, à une question portant sur le choix préférentiel d'un nouvel emploi, l'entreprise publique n'a été citée que par $25,9 \%$ des personnes interrogées. Les autres ont choisi le secteur non public : $16,7 \%$ souhaitant monter leur propre affaire et $3 \%$ voulant travailler à leur compte sous licence. Parmi les personnes qui étaient prêtes à travailler à risque (sans salaire garanti), $70 \%$ des hommes et $60 \%$ des femmes préféraient être employés par un entrepreneur privé. Environ $70 \%$ des sondés ont répondu par l'affirmative à la question de savoir si l'entrepreneur pouvait être désigné sous le terme « travailleur».

La formation dans le pays d'une couche d'entrepreneurs et de managers est une importante tendance observée ces dernières années. Leur état d'esprit a été révélé par une enquête distribuée par le Centre d'études sociales Rossika distribuée au cours d'une conférence de 600 représentants du « secteur alternatif » tenue au mois de février 1991.

Voici les réponses qui ont été données à la question : "Qu'est-ce qui gêne le plus l'instauration en URSS des rapports de marché ? » : la politique économique du gouvernement (55\%), le maintien au pouvoir de l'appareil du PCUS $(50 \%)$, l'absence de l'institution de la propriété privée (46\%). Treize pourcent seulement ont mentionné l'hostilité de la population vis-à-vis de la richesse et de l'esprit d'entreprise; $18 \%$, le parasitisme traditionnel. À la différence des sociologues, le nouveau monde des affaires n'est pas enclin à accorder une grande signification aux survivances antimarché dans la psychologie collective. À la question concernant les délais d'établissement dans le pays de conditions normales pour l'entreprise, $8 \%$ des personnes interrogées ont répondu qu'il faudra 1-2 ans, $37 \%, 3-5$ ans, $22 \%, 6-10$ ans. Mais si d'importants changements économiques et politiques se produisent, ces délais peuvent être ramenés à 1-2 ans (34\%) ou 3-5 ans (39\%) $)^{2}$.

Les deux tiers des sondés considèrent que la principale motivation de leur activité est la " sensation de véritablement gérer », l'« aspiration à la liberté » $(57 \%)$ et, seulement en troisième position $(54 \%)$, l'« amélioration de la condition matérielle ». Il est caractéristique que l'éparpillement des opinions des sondés en fonction du niveau d'instruction, du statut et des affinités économiques ne dépasse pas 5 ou $6 \%$, ce qui atteste un rapprochement sensible des vues et des positions de ce groupe social.

2. À cet égard, on doit rappeler que les boursiers moscovites, les courtiers et de nombreux petits entrepreneurs ont pris une part directe à l'annihilation de la tentative de coup d'État du mois d'août. 
Apparemment, le domaine sujet à la « petite » privatisation - le commerce et les services - recèle des potentialités notables pour muscler la petite entreprise. Un récent sondage effectué auprès de 940 entreprises de commerce de détail de Moscou a montré que près de $90 \%$ des dirigeants et $62 \%$ des collectifs de travail saluent la privatisation. La moitié des collectifs de travail sont prêts dès aujourd'hui à acheter leur magasin, $35 \%$ espèrent le faire dans les deux à trois années à venir. Mais quelle est l'importance de ces potentialités à l'échelle du pays ? Pour ce qui est de cette question, les statistiques nous laissent dans l'ignorance.

Toutefois, la plus grande incertitude est liée à l'évolution de la situation politique. L' « espace économique » dans lequel l'URSS se transforme prend corps au cours d'une lutte tendue. Il est évident pour tout le monde que l'économie soviétique ne peut plus fonctionner sans une modernisation fondamentale, mais la question est de savoir quelle sera la répartition des rôles dans ce processus. La transformation, observée jusqu'à ces derniers temps, de pans entiers de la propriété publique en propriété de groupes élitaires dans le but de les soustraire à la véritable privatisation, réduit notablement les possibilités de développement de la petite entreprise, ce qui hypothèque aussi la création future dans le pays d'une démocratie économique (et politique) de type occidental. Dans le contexte soviétique, une solution alternative est possible en transférant la propriété aux collectifs de travail, mais elle suscite d'âpres discussions au sein même des réformateurs. Les prochains mois seront probablement décisifs quant au choix de la politique économique. Dans une grande mesure, ce choix déterminera également les perspectives du développement de la petite entreprise dans la période à venir. 
ANNEXE

Les PME manufacturières en URSS à la fin des années 1980 (estimation, \%)

\begin{tabular}{|l|c|c|c|c|}
\hline & $\begin{array}{c}\text { Dans } \\
\text { le produit } \\
\text { brut }\end{array}$ & $\begin{array}{c}\text { Dans } \\
\text { le nombre } \\
\text { des salariés }\end{array}$ & $\begin{array}{c}\text { Dans } \\
\text { le capital } \\
\text { fixe }\end{array}$ & $\begin{array}{c}\text { Dans } \\
\text { la consommation } \\
\text { de l'énergie }\end{array}$ \\
\hline A. Petites & 1,8 & 1,7 & 1,2 & 0,6 \\
Moyennes & 12,9 & 13,2 & 9,1 & 4,7 \\
B. Petites & 5,4 & 5,3 & 3,5 & 1,7 \\
\hline
\end{tabular}

A. Petites - jusqu'à 100 salariés, moyennes - 100-500 salariés.

B. Petites - jusqu'à 200 salariés.

Source : Malyje predprijatija ... (Matériaux de la conférence). vol. 1, p. 10.

\section{Bibliographie}

Malyje predprijatija kak faktor formirovanija i razvitija rynocnoj ekonomiki. (Les documents de la conférence, Moscou, 2-4.4.1991). vol. 1 (p. 111); vol. 2 (p. 211). - Ronéotypé.

Les matériaux de la presse :

Voprosy ekonomiki.

Kommersant.

Ekonomika i zizn'.

Eko.

Moskovskije novosti.

Delovoy mir.

Megapolis - Express.

Argumenty i fakty.

Malyj business.

Izvestija.

Business $i$ banki.

Birzevyje vedomosti.

Delovyje ludi.

Sojuz. 